\title{
A Case of Subclavian Artery Injury Due to Blunt Trauma Treated Successfully by Endovascular Repair
}

\author{
Masayoshi Nishina ${ }^{1}$, Satoru Morita ${ }^{2)}$, Takamitsu Masuda ${ }^{1)}$, Hiroyasu Suga' \\ Toshimitsu Yokoyama ${ }^{1)}$, Takayuki Sato ${ }^{1)}$ and Shunshi Nishikubo ${ }^{1)}$ \\ ${ }^{1)}$ Emergency and Critical care Center, Tokyo Women's Medical University Medical Center East \\ ${ }^{2)}$ Department of Radiology, Tokyo Women's Medical University Medical Center East
}

\begin{abstract}
Subclavian artery injury is rare, but can cause both limb-and life-threatening conditions by rupture into the thoracic cavity. We report the case of a 59 -yearold man with subclavian artery injury due to blunt trauma that was successfully treated by endovascular repair. Computed tomography showed a small aneurysm in the left subclavian artery close to the fractured left clavicle. We initially attempted conservative treatment because the aneurysm was small and signs of ischemia were absent. However, the aneurysm continued to increase in size and showed a risk of rupture into the thoracic cavity, which would have been life-threatening. A covered stent was placed 2 weeks after injury following a rapid increase in size of the aneurysm with relapsed pain. The aneurysm disappeared following stent placement and patency of the subclavian artery was preserved. Endovascular repair should be considered in the early phase after subclavian artery injury, even if the lesion is small.
\end{abstract}

Key words: subclavian artery injury, subclavian artery aneurysm, blunt trauma, endovascular repair

\section{Introduction}

Subclavian artery injury is rare, but can cause both limb-and life-threatening conditions due to rupture into the thoracic cavity ${ }^{1,2)}$. Endovascular techniques

Received: July 12, 2011/Accepted: March 1, 2012 Correspondence to: Masayoshi Nishina Tokyo Women's Medical University Medical Center East Emergency and Critical care Center, 2-1-10 Nishiogu, Arakawa-ku, Tokyo 116-8567, Japan have recently been used to successfully treat vascular injury ${ }^{3}$. We report herein a case of subclavian artery injury due to blunt trauma successfully treated by endovascular repair.

\section{Case report}

A 59-year-old man with retinitis pigmentosa was hit by a truck while walking with his guide dog. He was struck in the face and left shoulder. After being brought to a local hospital, and transferred to our institute.

On arrival, his consciousness was clear, heart rate was 96 beats/min, blood pressure was $120 / 70 \mathrm{mmHg}$ without any difference in laterality, and respiratory rate was 12 breaths/min. The left shoulder region showed marked swelling. A pulse was clearly palpable in the left radial artery.

Radiography on day 1 showed left clavicular fracture. Contrast-enhanced computed tomography on day 1 showed an aneurysm $(6 \times 6 \mathrm{~mm})$ of the left subclavian artery close to the fractured left clavicle (Fig. 1 ), and maxillary and mandibular fractures. Computed tomography on day 5 showed slight enlargement of the aneurysm $(9 \times 7 \mathrm{~mm})$ (Fig. 2$)$. The patient complained of sudden left shoulder pain on day 12 , and computed tomography showed rapid enlargement of the aneurysm $(14 \times 14 \mathrm{~mm})$ (Fig. 3 ).

After discussion with a radiologist and cardiovascular surgeon, we decided to perform endovascular repair with preparation for conversion to open surgery in the event of failure on day 13. Informed written consent was obtained from the patient. A covered stent made of expanded polytetrafluoroethylene (ePTFE) was inserted through the left brachial artery. The stent was placed in the left subclavian 


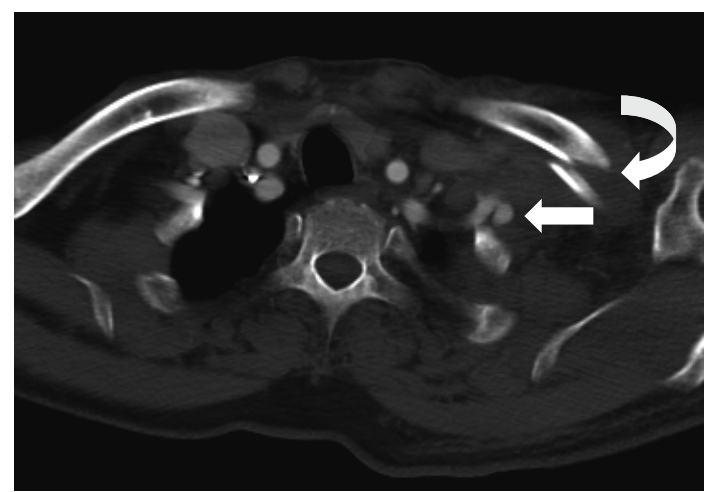

Fig. 1 Computed tomography on day 1.

Trans-axial image on contrast enhanced computed tomography on day 1 , showing aneurysm of the left subclavian artery $(6 \times 6 \mathrm{~mm}$, white straight arrow) and clavicular fracture (white curvilinear arrow).

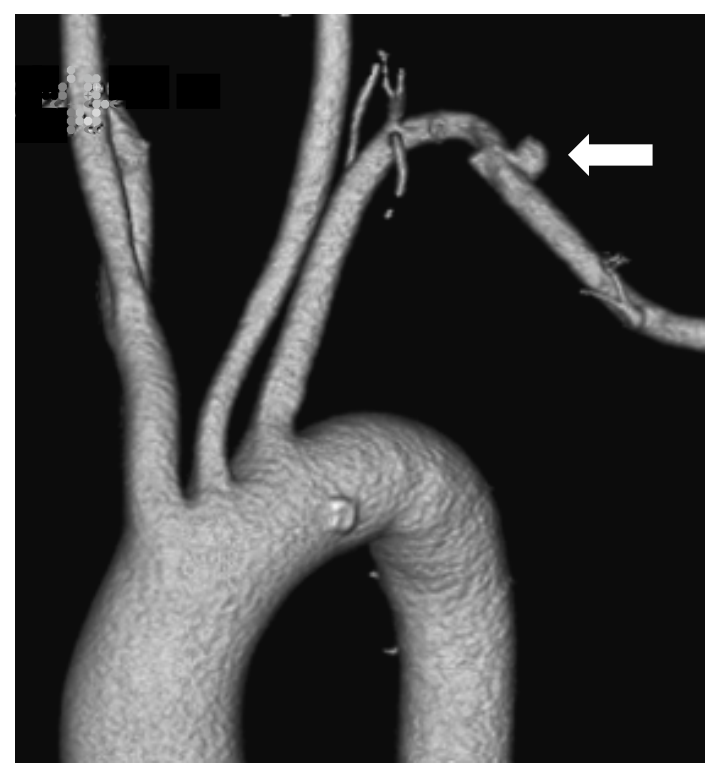

Fig. 2 Computed tomography on day 5.

Volume-rendered image of contrast-enhanced computed tomography on day 5 , showing slight enlargement of the aneurysm $(9 \times 7 \mathrm{~mm}$, white arrow).

artery just peripheral to the origins of the thyrocervical trunk and internal thoracic artery, fully peripheral to the origin of the vertebral artery (Fig. 4 ). No complications were observed during the procedure.

Aspirin and clopidogrel were administered as antiplatelet therapy. Computed tomography on day 16 showed that the stent graft was in a suitable position,

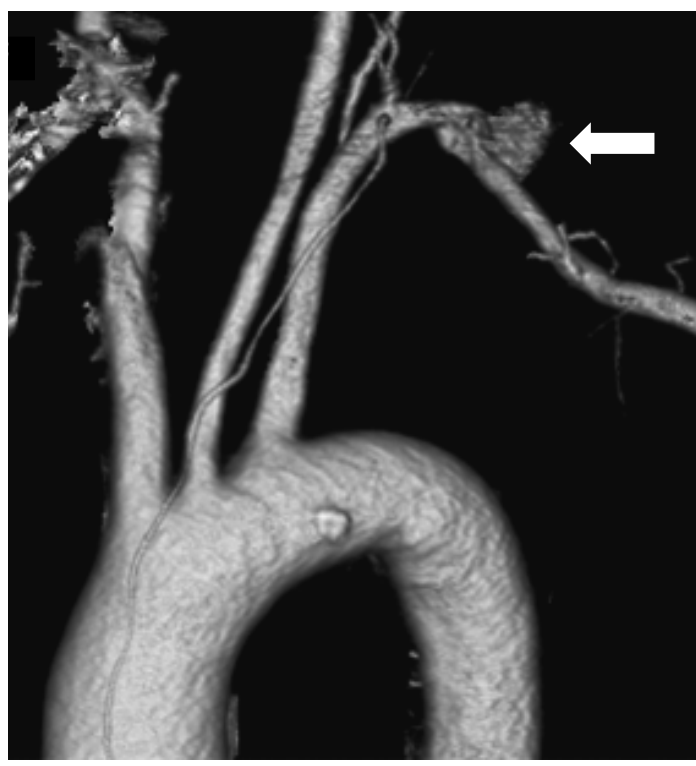

Fig. 3 Computed tomography on day 12 .

Volume-rendered image of contrast-enhanced computed tomography on day 12 , showing rapid enlargement of the aneurysm (14 $\times 14 \mathrm{~mm}$, white arrow).

and the aneurysm had disappeared (Fig. 5 ). The patient was transferred back to the previous hospital on day 19 , and was discharged on day 60 . With administration of aspirin and clopidogrel, no complaints of stenosis had been reported as of 15 months postoperatively.

\section{Discussion}

Subclavian artery injury is rare, accounting for only $1-2 \%$ of all vascular injuries ${ }^{1)}$, and $0.2 \%$ of all trauma center admissions ${ }^{1}$. The majority of subclavian artery injuries occur with penetrating trauma, with $1-5 \%$ resulting from blunt mechanisms ${ }^{1,2)}$. Blunt subclavian artery injuries are less common in the proximal portion of the artery than in the middle or distal portions ${ }^{1)}$. Vierhout et $\mathrm{al}^{3}$. reviewed 381 cases of subclavian artery aneurysm in the literature, finding trauma as the cause in 128 cases, and causes such as atherosclerosis or iatrogenic factors in the remaining cases.

Subclavian artery injuries after blunt trauma have not been characterized. The symptoms of subclavian artery aneurysm are shoulder pain, chest pain or pulsating mass ${ }^{3)}$. Mass effects can cause dysphagia, dyspnea or hoarseness. Rupture may prove fatal, or 

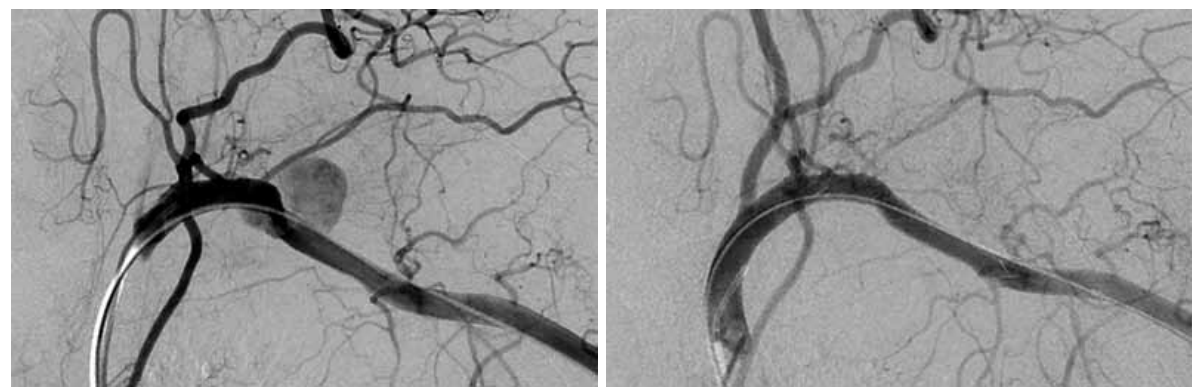

Fig. 4 Angiography on day 13.

Digital-subtraction angiography before stent insertion, showing the aneurysm (left). Digital subtraction angiography after stent insertion shows the aneurysm has disappeared (right).

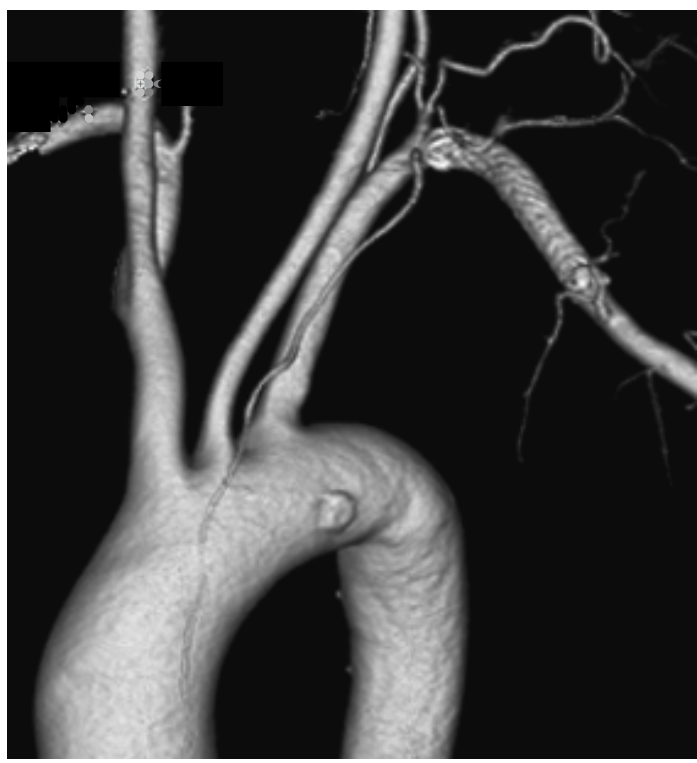

Fig. 5 Computed tomography on day 16 .

Volume-rendered image of contrast-enhanced computed tomography on day 16 , showing that the aneurysm has disappeared, while the thyrocervical trunk, internal thoracic artery and left vertebral artery remain preserved.

necessitate amputation.

The diagnosis of aneurysm located in the proximal segment of the subclavian artery is often difficult. In the present case, contrast-enhanced computed tomography was useful to confirm the diagnosis of subclavian artery aneurysm.

Options for the treatment of the subclavian artery aneurysm include conservative observation, open operation, and endovascular repair. We initially at- tempted conservative treatment because the aneurysm was small and signs of ischemia were absent. However, the aneurysm continued to increase in size and showed a risk of rupture into the thoracic cavity, which would have been life-threatening.

Surgical exposure of the subclavian artery injury may require sternotomy, thoracotomy, or clavicular transaction, whereas endovascular repair is less invasive. Recent endovascular techniques have been used successfully against vascular injury ${ }^{4-11)}$. Some endovascular procedures for injuries or aneurysms of the subclavian artery have been reported. However, reports of stent-graft repair to the subclavian artery aneurysm following blunt trauma are $\operatorname{rare}^{12-14)}$

Stent insertion alone is sometimes inadequate to treat the injury, and combinations of stenting with coils $^{15)}$, open surgery ${ }^{16)}$ or balloon occlusion ${ }^{14)}$ have been reported.

One limitation of endovascular treatment in this case was that although the membrane of the covered stent was made using ePTFE, which is commonly used for artificial blood vessels, long-term patency has not been confirmed. Furthermore, a covered stent has the potential for stent fracture, because the subclavian artery moves easily. We therefore fully informed the patient before treatment, and instructed him that if clinical findings of ischemia of the left upper limb appeared, he should immediately visit our hospital to undergo thrombolytic therapy or bypass surgery. Fortunately, no such findings have been observed in the 15 months since stent placement. 


\section{References}

1) Cox CS, Allen GS, Fischer RP, et al : Blunt versus penetrating subclavian artery injury : presentation, injury pattern, and outcome. J Trauma $46: 445-449$, 1999

2) Katras T, Baltazar U, Rush DS, et al : Subclavian arterial injury associated with blunt trauma. Vasc Surg 35:43-50, 2001

3) Vierhout BP, Zeebregts CJ, van den Dungen JJAM, et al : Changing profiles of diagnostic and treatment options in subclavian artery aneurysms. Eur J Vasc Endosc Surg $40: 27-34,2010$

4) Carrick MW, Morrison CA, Pham HQ, et al : Modern management of traumatic subclavian artery injuries : a single institution's experience in the evolution of endovascular repair. Am J Surg 199 : 28-34, 2010

5) Xenos ES, Freeman M, Stevens S, et al : Covered stents for injuries of subclavian and axillary arteries. J Vasc Surg $38: 451-454,2003$

6) Castelli P, Caronno R, Piffaretti G, et al : Endovascular repair of traumatic injuries of the subclavian and axillary arteries. Injury $36: 778-782,2005$

7) Hilfiker PR, Razavi MK, Kee ST, et al : Stent-graft therapy for subclavian artery aneurysms and fistulas : single-center mid-term results. JVIR $11: 578-584$, 2000

8) du Toit DF, Lambrechts AV, Stark H, et al : Longterm results of stent graft treatment of subclavian artery injuries : management of choice for stable pa- tients? J Vasc Surg $47: 739-743,2008$

9) Franz RW : Delayed treatment of a traumatic left subclavian artery pseudoaneurysm. Vasc Endovasc Surg $42:$ 482-485, 2008

10) Taha MM, Nakahara I, Higashi T, et al : Interventional neuroradiological techniques for the treatment of aneurysms of the supra-aortic extracranial arteries. Neurol Med Chir $50: 275-280,2010$

11) Carrafiello G, Lagana D, Mangini M, et al : Percutaneous treatment of traumatic upper-extremity arterial injuries : a single-center experience. J Vasc Interv Radiol $22: 34-39,2010$

12) Stockinger ZT, Townsend MC, McSwain NE, et al : Acute endovascular management of a subclavian artery injury. J La State Med Soc 156 : 262-264, 2004

13) Bukhari HA, Saadia R, Hardy BW : Urgent endovascular stenting of subclavian artery pseudoaneurysm caused by seatbelt injury. Can J Surg 50 : 303-304, 2007

14) Karkos CD, Mair R, Markose G, et al : Hybrid procedures combining open and endovascular surgical techniques for the management of subclavian artery injuries. J Trauma 63 : E107-E110, 2007

15) Babatani G, Massetti M, Le Page O, et al : Endovascular treatment of a traumatic subclavian artery aneurysm. J Trauma $44: 545-547,1998$

16) Bruen KJ, Freezor RJ, Lee WA : Hybrid management of proximal right subclavian artery aneurysms. J Vasc Surg $53: 528-530,2010$ 